\title{
Measurements of the magnetic field in WD $1658+441$
}

\author{
J. Ramírez Vélez ${ }^{1}$, D. Hiriart ${ }^{1}$, G. Valyavin ${ }^{2}$, J.Valdez $^{1}$, F. Quiroz ${ }^{1}$, \\ B. Martínez ${ }^{1}$, S. Plachinda ${ }^{2}$ and E. Iñiguez-Garín ${ }^{1}$ \\ ${ }^{1}$ Instituto de Astronomía, Universidad Nacional Autónoma de México. \\ Km. 103 Carretera Tijuana Ensenada, CP 22860, México. email: julio@astrosen. unam.mx \\ ${ }^{2}$ Special Astrophysical Observatory, Nizhnij Arkhyz, Karachai-Cherkessian, Russia 369167
}

\begin{abstract}
We present the preliminary results of the measurements of longitudinal magnetic field of the massive white dwarf $1658+441$. This star have an hydrogen pure atmosphere (e.g. Dupuis \& Chayer, 2003). We have observed the target in a total of $18 \mathrm{hrs}$ during 3 consecutive nights in June 2010 and one more in May 2011. The data was acquired with a prototypical spectropolarimeter at the San Pedro Martir Telescope in Mexico. We have tested the magnetic field measurements with our instrument using the famous Babcock's star obtaining consistent results with previous studies. For our object of study, the WD 1658+441, we have measured variable intensities of the longitudinal magnetic field of $B_{\text {long }}=720 \mathrm{kG}$ that oscillates with an amplitude of $130 \mathrm{kG}$.
\end{abstract}

Keywords. WD1658+441, HD215441, stellar magnetic fields, spectropolarimetry.

\section{Introduction}

The DA white dwarf $1658+441$ is a hot $\left(T_{\text {eff }}=30,000 K\right.$; Archilleos \& Wickrasmassinghe 1989), ultra massive star $\left(1.3 M_{\odot}\right)$, which host an intense variable magnetic field (Liebert et al., 1983).

We have chose this study object because is a suitable target to be observed with the prototypical spectropolarimeter at the 2.1-meters Telescope at the national observatory in "San Pedro Martir", Mexico.

We have performed a minimum of changes to the system Telescope-Spectrograph in order to use it as spectro-polarimeter. Before the slit of the low resolution spectrograph Boller \& Chivens, from 150 to 1200 grooves $/ \mathrm{mm}$, we have included a polarimetric analyzer module that includes a rotable quarter wave plate and a Savart plate. This configuration allow us to mesure the circular polarized spectra.

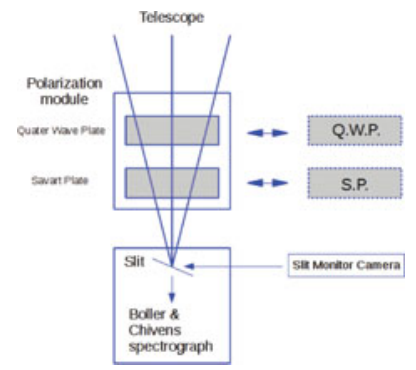

Figure 1. Optical design of the system Telescope - Polarizer Module - Spectrograph 

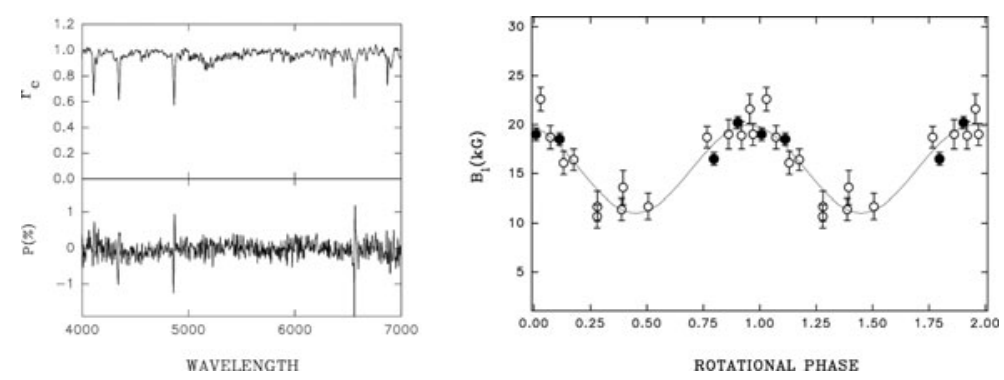

Figure 2. Left; spectra in intensity and circular polarization of HD215441. Right; magnetic field mesurements in function of the rotational phase of the star (see text for a description).
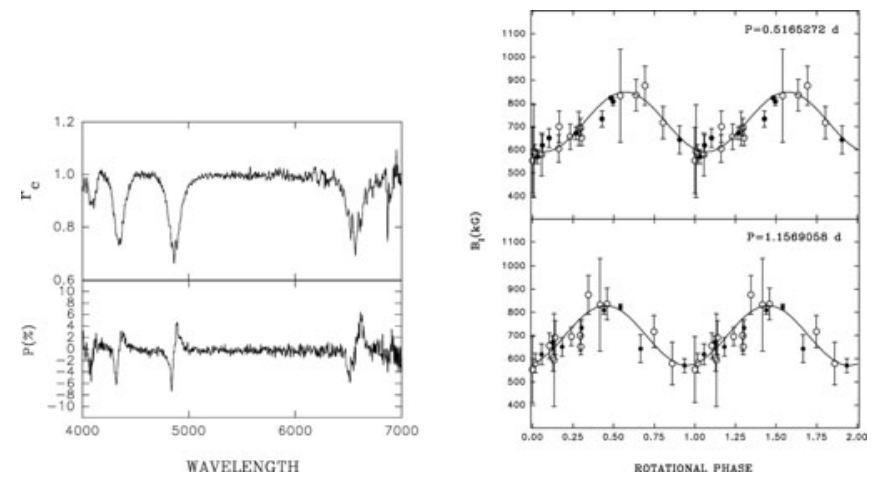

Figure 3. Left; spectra in intensity and circular polarization of WD1658+441. Right; magnetic field mesurements in function of the rotational phase of the star (see text for a description).

\section{Results}

In the left panel in Fig. 3, we show an example of the spectra of HD215441 which clearly shows the typical S-shape of the V-Stokes profile. Our measurements of the magnetic field are consistent with the values reported previously. In the right panel, the open circles correspond to Borra \& Landstreet (1981), while solid circles correspond to our data.

In Fig. 4, left panel, we show an example of observed spectra of our object of study, the WD1658+441. In the right panel, we have combined our magnetic fields measurements (solid circles) with those of Shtol' et al. (1997) (open circles) to analyze the periodicity of the field variations. The power spectra showed two main frequency peaks at 0.5 days and 1.6 days. We interpret these values as the range values for the most probable rotation period of the star. As conclusion, we found that the longitudinal magnetic field intensity of WD1658+441 varies with an amplitud of $130 \mathrm{kG}$ around a value of $720 \mathrm{kG}$.

Acknowledgements: This study is supported by CONACyT grant number 180817 . The authors thank to grant offered by the IAU-Symposium.

\section{References}

Achilleos, N. \& Wickramasinghe, D. T., 1989 PASA, 8, 148-153

Dupuis, J. \& Chayer, P., 2003, ApJ, 598, 486-491

Borra, A. \& Landstreet, J. D., 1981, ApJS, 264, 262-272

Liebert, J., Schmidt, G., Green, R., Bychkov, V. \& Stolyarov, V., 1997, AstL, 23, 48S

Shtol, V., Valyavin, G., Fabrica, S., Stockman, H. \& McGraw, J. 1983, ApJ, 264, 262-272 